\title{
Magnetic resonance imaging of skeletal muscle involvement in limb restricted vasculitis
}

\author{
S Gallien, A Mahr, F Réty, M Kambouchner, F Lhote, P Cohen, L Guillevin
}

Ann Rheum Dis 2002;61:1107-1 109

Background: Limb restricted polyarteritis nodosa (PAN) and PAN-type diseases such as isolated vasculitis of skeletal muscle are localised vasculitides affecting the skin, muscles, or peripheral nerves, usually of the lower limbs. These diseases often present with non-specific clinical symptoms and normal laboratory values and electromyograms. The usefulness of magnetic resonance imaging (MRI) of skeletal muscle has been poorly investigated to date.

Objective: To describe the MRI findings in the legs of three patients with limb restricted vasculitides (two PAN, one isolated vasculitis of the skeletal muscle) with histologically established muscle involvement.

Methods: MRI was carried out on calf muscles and $\mathrm{T}_{2}$ weighted images, unenhanced $T_{1}$ weighted images, and STIR sequences were obtained.

Results: Muscle damage resulted in oedema-like changes on MRI characterised by hyperintense signals in $\mathrm{T}_{2}$ weighted and slow tau inversion recovery (STIR) sequences and normal unenhanced $T_{1}$ weighted sequences of one or several leg muscles.

Conclusions: MRI should be considered a useful complementary examination that might facilitate the recognition of limb restricted vasculitides, and possibly indicate the site for muscle biopsy. It could also be useful in monitoring the course of the disease. Future studies should also evaluate MRI for systemic PAN or other systemic vasculitides with muscle involvement.

$\mathrm{P}$ olyarteritis nodosa (PAN) is a necrotising vasculitis predominantly involving medium sized arteries that typically presents as a systemic disease. ${ }^{1}$ In a subset of patients with PAN, however, the disease process remains localised within a single organ or a precise anatomical area. The best known localised form of PAN is limb restricted vasculitis-mainly of the legs-which has been called cutaneous PAN..$^{2-4}$ Initially thought to be a cutaneous disease, ${ }^{5}$ this limb restricted form of PAN also commonly extends to the adjacent muscles, peripheral nerves, and joints. ${ }^{2}$ Subsequently, other PAN-like diseases of the limbs, involving exclusively skeletal muscle ${ }^{67}$ or peripheral nerves, ${ }^{8}{ }^{9}$ were reported. These limb restricted vasculitides have a good prognosis but an often chronic and relapsing course. ${ }^{39}$

The diagnosis of limb restricted vasculitis relies principally on the findings of a cutaneous, ${ }^{3}$ peripheral nerve, ${ }^{9}$ or muscle biopsy. $^{7}$ The usefulness of MRI studies of the affected muscle has been poorly investigated to date. ${ }^{10}$ Herein we describe three patients with limb restricted vasculitis (two with PAN, one with isolated vasculitis of skeletal muscle) for which MRI of the legs contributed substantially to the diagnostic investigation and disease management.

\section{CASE REPORTS}

\section{Patient 1}

A 40 year old man with no medical history of consequence was referred to our department in December 1998 because of a three month history of painful induration of the external face of the left calf and arthralgia of the left ankle.

Physical examination found no fever, a blood pressure of $140 / 80 \mathrm{~mm} \mathrm{Hg}$, and a palpable tumour about $2 \mathrm{~cm}$ in diameter on the external face of the left calf. Laboratory data showed an erythrocyte sedimentation rate (ESR) of $39 \mathrm{~mm} / \mathrm{lst} \mathrm{h}$. Creatine kinase (CK) and aldolase levels, liver function tests, and serum complement levels were normal, and there was no creatininaemia or proteinuria. Antibody tests for HIV and hepatitis $C$ virus were negative, and hepatitis B virus serology detected only anti-HBs antibodies. Tests for antinuclear antibodies (ANAs), antineutrophil cytoplasm antibodies (ANCAs) and cryoglobulins were negative, and a chest radiograph was normal. Magnetic resonance imaging of the calves showed normal unenhanced $T_{1}$ weighted sequences, and hyperintense signals on $\mathrm{T}_{2}$ weighted and slow tau inversion recovery (STIR) images of the peroneus longus, peroneus brevis, and tibialis anterior muscles of the left calf (fig 1); the same areas were slightly enhanced by gadolinium in $T_{1}$ weighted sequences. Histological examination of biopsy performed in the pathological area disclosed vasculitis of medium sized $(250 \mu \mathrm{m})$ arteries with fibrinoid necrosis of the vessel wall, and a polymorphous inflammatory infiltrate containing predominantly neutrophils and eosinophils. Limb restricted PAN-type vasculitis of skeletal muscle was diagnosed.

In January 1999, prednisone (40 mg/day) was started, and provided rapid relief of myalgia and arthralgia, but the symptoms recurred during dose tapering. Serial MRI of the calves showed persistent signal abnormalities of the left anterolateral compartment. In June 2000, azathioprine ( 100 mg/day) was added to the prednisone. One year later, the patient is still taking azathioprine and prednisone $(20 \mathrm{mg} /$ day) and has only moderate pain.

\section{Patient 2}

In June 1995, a previously healthy 41 year old woman was referred to our department for myalgia of the right calf, arthralgia of the right ankle of 10 months' duration, and subsequent development of bilateral skin nodules in the legs.

Temperature and blood pressure were normal, but there were multiple tender subcutaneous nodules on both lower limbs with slight inflammatory oedema and tenderness of the underlying muscles. Her ESR was $42 \mathrm{~mm} / \mathrm{lst}$ h. Values for CK,

Abbreviations: ANA, antinuclear antibodies; $A N C A$, antineutrophil cytoplasm antibodies; CK, creatine kinase; ESR, erythrocyte sedimentation rate; $\mathrm{HBV}$, hepatitis $B$ virus; $\mathrm{HCV}$, hepatitis $\mathrm{C}$ virus; MRI, magnetic resonance imaging; PAN, polyarteritis nodosa; RF, rheumatoid factor; STIR, slow tau inversion recovery 


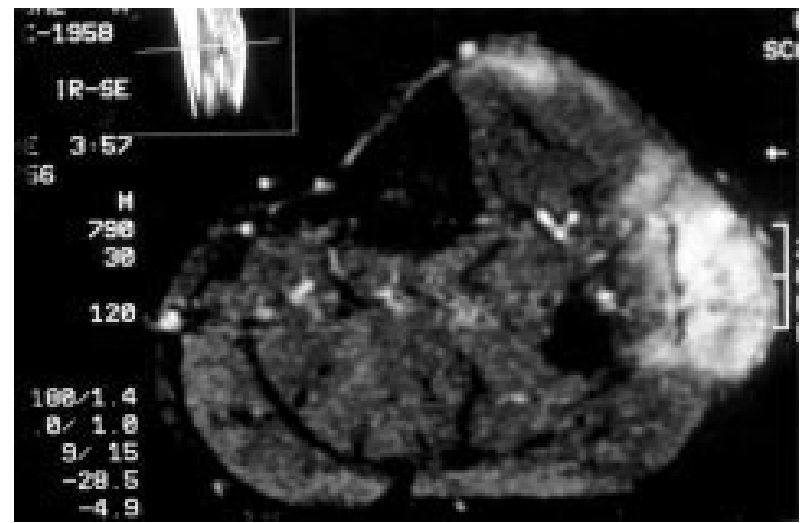

Figure $1 \mathrm{MRI}$ of the calves of a 40 year old man with PAN-type vasculitis confined to the leg muscles. The axial STIR image shows a patchy hyperintensity in the tibialis anterior and peroneus brevis muscles and a diffuse increase of the signal in the peroneus longus.

aldolase, and serum complement were normal and there was no creatininaemia Serological assays for hepatitis B virus (HBV) and hepatitis C virus (HCV), ANA, ANCA, rheumatoid factor (RF), and cryoglobulins were negative. Radiographs of the calves and a technetium-99m bone scan were consistent with bilateral but predominantly right fibular periostitis. Magnetic resonance imaging of the calves showed bilateral and patchy distributions of hyperintense signals on $\mathrm{T}_{2}$ weighted images of the anterolateral and posterior compartments. The involved area was enhanced by gadolinium in $T_{1}$ weighted sequences. Histological examinations of a muscle biopsy guided by MRI and an excised cutaneous nodule showed fibrinoid necrosis of a medium sized artery wall, an inflammatory cell infiltrate with lymphohistiocytes, and epithelioid giant cell granuloma. Lower limb restricted PAN involving skin and muscle and periosteal reaction was diagnosed.

Prednisone (50 mg/day) was started in September 1995. The symptoms regressed but myalgia recurred in October 1996 on $5 \mathrm{mg}$ prednisone a day. Colchicine ( $1 \mathrm{mg} / \mathrm{day}$ ) was prescribed and allowed transient discontinuation of prednisone. In June 1998, prednisone (10 mg/day) was reintroduced because of the reappearance of cutaneous lesions and was continued as maintenance treatment. In April 1999 a new disease flare up occurred with MRI of the calves showing the same abnormalities as previously.

\section{Patient 3}

In August 1998, a 45 year old woman was referred to our department because of an 11 month occurence of arthralgia of the ankles and severe myalgia with swelling of the calves. The patient had a history of chronic HCV infection (serotype 3) diagnosed in 1997. A needle biopsy of the liver performed in April 1998 was consistent with chronic mildly active hepatitis.

Physical examination showed a normal temperature, a blood pressure of $140 / 80 \mathrm{~mm} \mathrm{Hg}$, and bilateral tenderness with a slight, inflammatory oedema of the calves, which had several small sized erythematous macular lesions. Laboratory tests showed an ESR of $30 \mathrm{~mm} / 1 \mathrm{st} h$, and normal CK and lactate dehydrogenase values. Liver enzymes, a chest radiograph, and urinary sediment were normal and there was no creatininaemia or proteinuria. Serum HCV RNA was detected at 13000000 copies/ml. Serological assays for HBV, HIV, ANA, RF, ANCA, and cryoglobulins were negative. Magnetic resonance imaging of the calves showed normal $\mathrm{T}_{1}$ weighted sequences but hyperintensities on $\mathrm{T}_{2}$ weighted images of both gastrocnemius muscles, which appeared swollen (fig 2 A). A cutaneous lesion and the medial head of the left gastrocnemius muscle were biopsied. Both samples contained prominent multifocal vasculitis characterised by polymorphous,
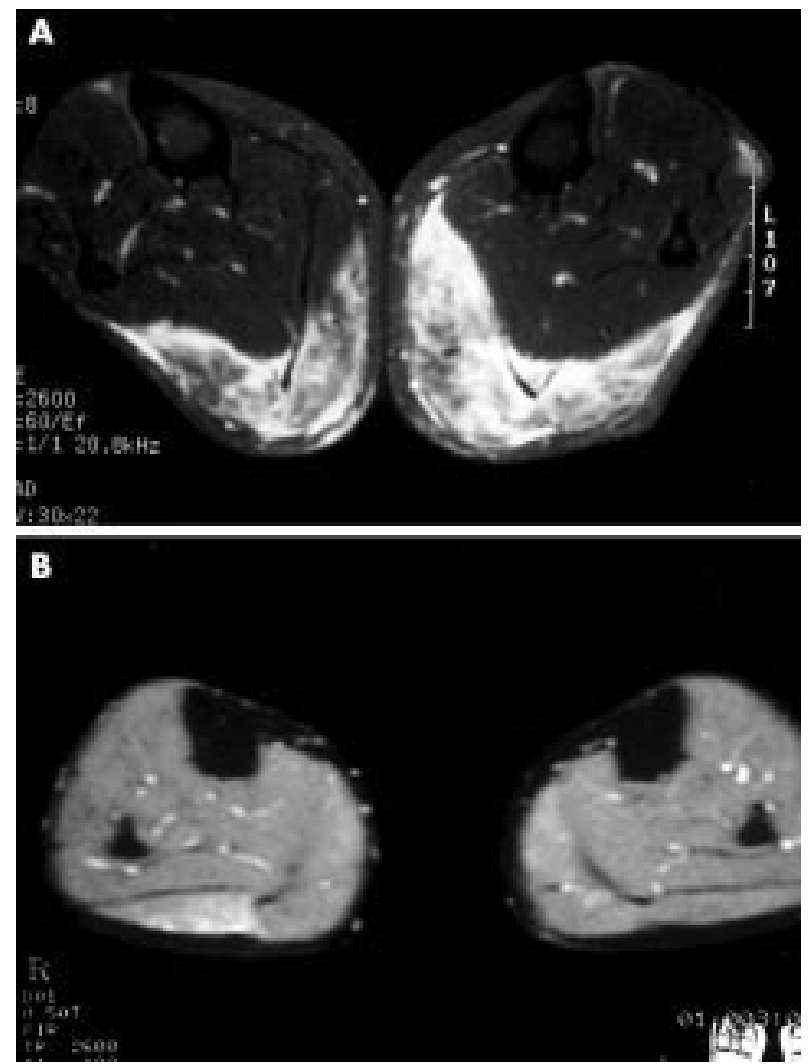

Figure 2 MRI of the calves of a 45 year old woman with limb restricted PAN associated with chronic HCV infection. Axial STIR sequences: $(A)$ initially, MRI detected areas of diffuse and symmetric hyperintensities in both gastrocnemius muscles and swelling of the affected muscles; (B) images taken 14 months after starting treatment show a normalisation of the hyperintense signals in the left gastrocnemius muscle and the medial head of the right gastrocnemius muscle. The lateral head of the left gastrocnemius muscle is still swollen and shows a mildly intense signal.

inflammatory infiltrates of medium sized arteries. Limb restricted PAN with skin and muscle involvement associated with chronic hepatitis $\mathrm{C}^{11}$ was diagnosed.

In September 1998, antiviral treatment with interferon-2 $\alpha$ and ribavirin was started. Because the myalgia did not regress, prednisone ( $40 \mathrm{mg} /$ day) was added in November 1998. Plasma exchanges and intravenous immunoglobulins remained ineffective. In June 1999, a monthly pulse of cyclophosphamide replaced intravenous immunoglobulins and led to a dramatic attenuation of the symptoms. Magnetic resonance imaging performed in October 1999 showed marked regression (fig 2 B) of the initial abnormalities.

\section{DISCUSSION}

Our findings in these three patients suggest that MRI can assess the muscle involvement occurring in PAN or PAN-type vasculitides restricted to the limbs. Indeed, muscle vasculitis appears as hypersignals in $\mathrm{T}_{2}$ weighted or STIR sequences. In $\mathrm{T}_{1}$ weighted sequences, the affected areas remained normal but were slightly enhanced by gadolinium. In all three patients, the hyperintensities involved one or several muscle groups of one or both lower limbs. These results are in accordance with those of Hofman et al, who reported the MRI findings in a patient with limb restricted PAN. ${ }^{10}$

Hyperintensities appearing predominantly or exclusively in $\mathrm{T}_{2}$ weighted or STIR sequences indicate increased muscle fluid content. Our patients' radiological findings are most likely consistent with oedema, which is the principal feature of muscle inflammation. ${ }^{12-14}$ However, because myopathies of 
traumatic, metabolic, and degenerative origin and peripheral neuropathies ${ }^{12-13} 15$ exhibit these same changes, some authors prefer the term oedema-like abnormalities rather than oedema. ${ }^{15}$ It is therefore unlikely that a diagnosis of limb restricted vasculitis may be evoked based solely on MRI findings. In particular, the patchy, asymmetric, and distal distribution of the lesions resembles that found in focal myositis. ${ }^{15}$

Despite an apparently poor specificity, MRI of skeletal muscle could constitute a useful complementary examination for limb restricted PAN or PAN-type vasculitides. These diseases are often difficult to recognise because of their indolent course and the poor specificity of clinical and laboratory signs. Myalgia and stiffness are the main clinical signs of muscle involvement. ${ }^{237}$ Levels of CK are normal or only slightly increased ${ }^{7}$ and electromyograms inconsistently show a myogenic pattern. ${ }^{2}$ Hence, our findings would indicate that MRI has a higher ability to detect vasculitic muscle disease. None the less, further investigation needs to be undertaken to evaluate the precise sensitivity of MRI in this setting. Another application of MRI could be to select the optimal site for muscle biopsy to ensure that the sampled tissue comes from an affected area, whereas serial MRI might be helpful for monitoring the course of the disease and response to treatment. As suggested by the normalisation of the oedemalike abnormalities of patient 3 under treatment and the patient reported by Hofman et al, ${ }^{10}$ it would seem that the MRI results coincide with disease activity, although these findings require confirmation.

It would also be informative to investigate the usefulness of skeletal muscle MRI for systemic PAN or other systemic vasculitides. In systemic PAN, skeletal muscles are often involved and represent one of the preferential biopsy sites for histological confirmation. ${ }^{1}$ The tecnique of MRI could therefore contribute to diagnosing paucisymptomatic disease or selecting the muscle biopsy site. It remains, however, to be shown that the muscle involvement occurring in systemic PAN generates the same changes in MRI as those seen in the more longstanding, limited disease.

\section{Authors' affiliations}

S Gallien, A Mahr, F Lhote, P Cohen, L Guillevin, Department of Internal Medicine, UPRES EA 3409 Hôpital Avicenne, 93009 Bobigny, France

F Réty, Department of Radiology, Hôpital Avicenne

M Kambouchner Department of Pathology, Hôpital Avicenne

Correspondence to: Dr L Guillevin, Department of Internal Medicine, 125 Route de Stalingrad, 93009 Bobigny, France;

loic.guillevin@avc.ap-hop-paris.fr

Accepted 8 May 2002

\section{REFERENCES}

1 Lhote F, Guillevin L. Polyarteritis nodosa, microscopic polyangiitis, and Churg-Strauss syndrome. Clinical aspects and treatment. Rheum Dis Clin North Am 1995;21:911-47.

2 Diaz-Perez JL, Winkelmann RK. Cutaneous periarteritis nodosa. Arch Dermatol 1974;1 10:407-14.

3 Moreland LW, Ball GV. Cutaneous polyarteritis nodosa. Am J Med 1990;88:426-30.

4 Borrie P. Cutaneous polyarteritis nodosa. Br J Dermatol 1972;87:87-95.

5 Lindberg K. Ein Beitrag zur Kenntnis der Periarteritis Nodosa. Acta Scand Med 1931;76:183-225.

6 Golding DN. Polyarteritis presenting with leg pains. BM 1970;i:277-8.

7 Garcia F, Pedrol E, Casademont J, Mellado B, Cordoba R, Cid M, et al. Polyarteritis nodosa confined to calf muscles. J Rheumatol 1992; 19:303-5.

8 Dyck PJ, Benstead TJ, Conn DL, Stevens JC, Windebank AJ, Low PA Nonsystemic vasculitic neuropathy. Brain 1987;1 10:843-53.

9 Davies L, Spies JM, Pollard JD, McLeod JG. Vasculitis confined to peripheral nerves. Brain 1996;1 19:1441-8.

10 Hofman DM, Lems WF, Witkamp TD, Putte VD, Biilsma JW. Demonstration of calf abnormalities by magnetic resonance imaging in polyarteritis nodosa. Clin Rheumatol 1992;11:402-4.

11 Soufir N, Descamps V, Crickx B, Thibault V, Cosnes A, Becherel PA, et al. Hepatitis $C$ virus infection in cutaneous polyarteritis nodosa: a retrospective study of 16 cases. Arch Dermatol 1999:135:1001-2.

12 Reimers CD, Finkenstaedt M. Muscle imaging in inflammatory myopathies. Curr Opin Rheumatol 1997;9:475-85.

13 Adams EM, Chow CK, Premkumar A, Plotz PH. The idiopathic inflammatory myopathies: spectrum of MR imaging findings. Radiographics 1995; 15:563-74.

14 Fleckenstein JL, Reimers CD. Inflammatory myopathies: radiologic evaluation. Radiol Clin North Am 1996;34:427-39.

15 Reimers CD, Schedel H, Fleckenstein JL, Nagele M, Witt TN, Pongratz $\mathrm{DE}$, et al. Magnetic resonance imaging of skeletal muscles in idiopathic inflammatory myopathies of adults. J Neurol 1994;241:306-14. 